\title{
Efficacy of neurointervention combined with intravenous thrombolysis in the treatment of ischemic cerebrovascular disease and its influence on neurological function and prognosis of patients
}

\author{
SHIXING LI, XUELEI LOU, ZUKUAN CHANG, CHAOHENG SHI, HUILIN LU and JIANKUI HAN \\ Department of Interventional Therapy, Xinxiang Central Hospital, Xinxiang, Henan 453000, P.R. China
}

Received June 17, 2019; Accepted July 21, 2020

DOI: $10.3892 /$ etm.2020.9404

\begin{abstract}
The present study aimed to investigate the efficacy of neurointervention combined with intravenous thrombolysis in the treatment of ischemic cerebrovascular disease (ICD) and its influence on the neurological function and prognosis of patients. A total of 119 patients with ICD admitted to Xinxiang Central Hospital (Xinxiang, China) from May 2013 to September 2015 were selected. Among them, 65 patients were enrolled in the control group and treated with intravenous thrombolysis, whereas the other 54 patients were enrolled in the observation group and were treated with intravenous thrombolysis combined with neurointervention. The National Institute of Health Stroke Scale (NIHSS) system was used to evaluate the neurological function of patients after treatment. Kaplan-Meier survival curve analysis was carried out to assess the survival of patients. The total effective rate, complications, vascular recanalization and the hospitalization time after treatment were compared between the two groups. The NIHSS scores at 1, 3 and 6 months after treatment were statistically lower in the observation group than those in the control group $(\mathrm{P}<0.05)$. The total effective rate and total vascular recanalization in the observation group were higher than those of the control group $(\mathrm{P}<0.05)$. The incidence of complications in the observation group was statistically lower than that in the control group $(\mathrm{P}<0.05)$. In conclusion, neurointervention combined with intravenous thrombolysis can not only effectively improve the diseased blood vessels of patients and restore the damaged nerve function, but also reduce the incidence of complications. Moreover, neurointervention combined with intravenous thrombolysis is safe and can ensure a better quality of life of patients.
\end{abstract}

Correspondence to: Dr Jiankui Han, Department of Interventional Therapy, Xinxiang Central Hospital, 56 Jinsui Avenue, Weibin, Xinxiang, Henan 453000, P.R. China

E-mail: jhnd53@163.com

Key words: neurointervention, intravenous thrombolysis, ischemic cerebrovascular disease, National Institute of Health Stroke Scale, efficacy

\section{Introduction}

Despite the improved living standards nowadays, ischemic cerebrovascular disease (ICD), a disease prevalent in the elderly, is quite common in this increasingly aging society. Featuring high mortality, recurrence and high disability rate, ICD poses a serious threat to people's lives (1-3). ICD can cause temporary blood supply disorder, which leads to neurological deficits and has a serious impact on patients (4). ICD onset is often accompanied by neurological dysfunction (5). Timely treatment is unavailable due to the sudden onset of ICD, making the life of patients severely impacted (6).

The prominent clinical treatment for ICD is intravenous thrombolysis, which can promote the recanalization of the blocked blood vessels and save the life of patients, because of the plasminogen activator in the thrombolytic drug which dissolves the fibrin in the thrombus (7-9). However, the deficiency is that intravenous thrombolysis only works within the first $4 \mathrm{~h}$ after the onset of the disease and has certain contraindications for patients (10). Therefore, only a limited number of patients are suitable for intravenous thrombolysis. Due to the increasing incidence of ICD, new research and discoveries have been made. In recent years, neurointervention has been applied in the treatment of ICD (11). Neurointervention is a minimally invasive therapy that is based on a computer to determine the location of arterial stenosis and treat cerebrovascular disease through intubation. The use of intravenous thrombolysis in the course of interventional therapy can directly unblock blood vessels and eliminate infarction $(12,13)$. Neurointervention compensates for the limitations of intravenous thrombolysis regarding treatment time and eligibility of patients. Therefore, the aim of the present study was to combine the two therapies in order to investigate whether intravenous thrombolysis combined with neurointervention could improve the ICD treatment.

The present study explored the efficacy of neurointervention combined with intravenous thrombolysis in the treatment of ICD and its influence on neurological function and prognosis of patients, aiming to provide future reference for the treatment of ICD.

\section{Patients and methods}

General information. A total of 119 patients with ICD admitted to Xinxiang Central Hospital (Xinxiang, China) 
from May 2013 to September 2015 were selected. Among them, 65 patients were enrolled in the control group and treated with intravenous thrombolysis, whereas the other 54 patients were enrolled in the observation group and were treated with intravenous thrombolysis combined with neurointervention. The control group included 37 males and 28 females, with an average age of $41.3 \pm 5.2$ years. In the control group, 18 patients had total anterior circulation infarction, 16 partial anterior circulation infarction, 19 posterior circulation infarction and 12 lacunar infarction. The observation group included 28 males and 26 females, with an average age of $42.3 \pm 4.7$ years. In the observation group, 15 patients had total anterior circulation infarction, 11 partial anterior circulation infarction, 17 posterior circulation infarction and 11 lacunar infarction. The study was approved by the Ethics Committee of Xinxiang Central Hospital. Patients who participated in the study had complete clinical data and signed written informed consents were obtained from the patients and/or guardians.

Inclusion and exclusion criteria. The inclusion criteria were the following: patients with ICD for the first time; patients with treatment indications for neurointervention; patients admitted to the hospital within $6 \mathrm{~h}$ after the ICD onset; patients diagnosed with ICD by head CT angiography; and patients with complete clinical data.

The exclusion criteria were the following: patients with severe inflammation; patients with severe mental disorder; patients with intracranial hemorrhage; patients with hereditary diseases; patients with malignant tumors; and patients with other serious cardiovascular or cerebrovascular diseases.

Treatment methods. Patients in the control group were treated with intravenous thrombolysis. After admission, patients were treated with tissue-type plasminogen activator for injection (China FDA approval no. S20150001; Guangzhou Recomgen Biotech Co., Ltd.). An intravenous drop of 300,000-500,000 U of urokinase and $20 \mathrm{ml}$ of saline was firstly administered, followed by an intravenous injection of 1,000,000 $\mathrm{U}$ of urokinase and $100 \mathrm{ml}$ of saline.

In the observation group, patients received neurointervention treatment right after the intravenous thrombolysis. Electrocardiography, blood routine tests, liver and kidney function tests, as well as coagulation function tests were performed before treatment. Aspirin (China FDA approval no. H41021076; Henan Fusen Pharmaceutical Co., Ltd.) was orally administrated at a dose of $50-150 \mathrm{mg} / \mathrm{day}$ and clopidogrel [China FDA approval no. 20180029; Sinofi (Hangzhou) Pharmaceutical Co., Ltd.] was orally administrated at a dose of $75 \mathrm{mg} /$ day to prevent platelet aggregation. With the support of the digital subtraction angiography system, an injection of a small amount of anesthetic into the inguinal region was administered before puncturing the femoral artery to place the vascular sheath. A $5 \mathrm{~F}$ arterial sheath was inserted. A contrast tube was inserted to the aortic arch under the guidance of the ultra-sliding guidewire. The blood vessel direction and position was confirmed by angiography. A total of 200,000 U of urokinase (national medicine standard $\mathrm{H} 22023486$ ) was added into $20 \mathrm{ml}$ of $0.9 \%$ sodium chloride solution for injection. Next, the solution was pumped in at a rate of $1 \mathrm{ml} / \mathrm{min}$ through a micropump autolysis catheter under the guidance of the
Table I. Efficacy evaluation criteria.

\begin{tabular}{ll}
\hline Items & \multicolumn{1}{c}{ Criteria } \\
\hline Marked response & $\geq 45 \%$ decrease in NIHSS score \\
Moderate response & $<45 \%$ and $\geq 18 \%$ decrease in \\
& NIHSS score \\
No response & $<18 \%$ decrease in NIHSS score
\end{tabular}

Table II. Criteria for vascular recanalization.

\begin{tabular}{ll}
\hline Items & \multicolumn{1}{c}{ Criteria } \\
\hline $\begin{array}{l}\text { Complete } \\
\text { recanalization }\end{array}$ & $\begin{array}{l}\text { The main branches of the skull are } \\
\text { completely recanalized and can be } \\
\text { clearly developed } \\
\text { Partial recanalization }\end{array}$ \\
$\begin{array}{l}\text { The main branches of the skull are } \\
\text { not clearly developed and the blood } \\
\text { vessels in the posterior part of the } \\
\text { embolization are partially recanalized } \\
\text { The blood vessels at the back end } \\
\text { of the embolization site are blocked } \\
\text { and cannot be developed }\end{array}$ \\
\hline
\end{tabular}

micro guidewire. A stent was placed at the site of arterial stenosis to determine the unclogging of blocked blood vessels. Antiplatelet therapy was performed $24 \mathrm{~h}$ after surgery.

Outcome measures. Main outcome measures: The National Institutes of Health Stroke Scale (NIHSS) (14) scores of the two groups of patients at 1,3 and 6 months after treatment were recorded. Kaplan-Meier survival curves were drawn to assess the patients' survival 1 year after treatment.

Secondary outcome measures: The efficacy of treatment (Table I) was evaluated in the two groups. Total effective rate $=($ cases of marked response + cases of moderate response)/total no. of cases x $100 \%$. In addition, the vascular recanalization rates (Table II) of the two groups were calculated. Vascular recanalization rate $=($ cases of complete recanalization + cases of partial recanalization)/total no. of cases $\times 100 \%$.

Statistical analysis. Statistical analysis was performed using SPSS 19.0 software (IBM Corp.) and the data were plotted using GraphPad Prism 7 software (GraphPad Software, Inc.). Count data were expressed as rate $(\%)$ and were compared by Chi-square test. Measurement data were expressed as the mean \pm standard deviation (mean \pm SD) and were compared between two groups by the independent samples t-test. The measurement data were normally distributed. Repeated measures ANOVA, followed by Bonferroni post hoc test, was used for the comparison of the data at multiple time points. The 1-year survival of patients was assessed by Kaplan-Meier survival analysis and log-rank test was used for the comparison of the survival curves. $\mathrm{P}<0.05$ was considered to indicate a statistically significant difference. 
Table III. General clinical data of patients in the control and observation groups [n (\%), mean \pm SD].

\begin{tabular}{|c|c|c|c|c|}
\hline Factors & Observation group $(n=54)$ & Control group $(n=65)$ & $t / \chi^{2}$ & P-value \\
\hline Sex & & & 0.306 & 0.580 \\
\hline Male & $28(51.85)$ & $37(56.92)$ & & \\
\hline Female & $26(48.15)$ & $28(43.08)$ & & \\
\hline Age (years) & $42.3 \pm 4.7$ & $41.3 \pm 5.2$ & 1.026 & 0.307 \\
\hline Disease type & & & 0.681 & 0.496 \\
\hline Total anterior circulation infarction & $15(27.78)$ & $18(27.69)$ & & \\
\hline Partial anterior circulation infarction & $11(20.37)$ & $16(24.62)$ & & \\
\hline Posterior circulation infarction & $17(31.48)$ & $19(29.23)$ & & \\
\hline Lacunar infarction & $11(20.37)$ & $12(18.46)$ & & \\
\hline Low-density lipoprotein $(\mu \mathrm{mol} / \mathrm{l})$ & $2.89 \pm 0.87$ & $2.81 \pm 0.79$ & 1.149 & 0.251 \\
\hline Hematological disease & & & 0.563 & 0.453 \\
\hline Yes & $18(33.33)$ & $26(40.00)$ & & \\
\hline No & $36(66.67)$ & $39(60.00)$ & & \\
\hline Fibrinogen $(\mathrm{g} / \mathrm{l})$ & $6.43 \pm 1.25$ & $6.26 \pm 1.43$ & 0.683 & 0.496 \\
\hline Triglyceride (mmol/l) & $1.35 \pm 0.23$ & $1.34 \pm 0.34$ & 0.184 & 0.854 \\
\hline Total cholesterol $(\mu \mathrm{mol} / \mathrm{l})$ & $4.49 \pm 1.04$ & $4.48 \pm 1.09$ & 0.051 & 0.960 \\
\hline Alcohol abuse & & & 0.351 & 0.553 \\
\hline Yes & $22(40.74)$ & $30(46.15)$ & & \\
\hline No & $32(59.26)$ & $35(53.85)$ & & \\
\hline
\end{tabular}

Table IV. Comparison of neurological deficits (NIHSS scores) between the two groups before and after treatment (mean \pm SD).

\begin{tabular}{|c|c|c|c|c|c|c|}
\hline Group & $\begin{array}{c}\text { Before } \\
\text { treatment }\end{array}$ & $\begin{array}{c}1 \text { month } \\
\text { after treatment }\end{array}$ & $\begin{array}{c}3 \text { months } \\
\text { after treatment }\end{array}$ & $\begin{array}{c}6 \text { months } \\
\text { after treatment }\end{array}$ & $\mathrm{F}$ & P-value \\
\hline Control group $(n=65)$ & $12.76 \pm 2.85$ & $10.02 \pm 2.91^{\mathrm{a}}$ & $9.24 \pm 2.32^{\mathrm{a}}$ & $8.32 \pm 2.16^{\mathrm{a}, \mathrm{b}}$ & 36.259 & $<0.001$ \\
\hline Observation group $(n=54)$ & $12.83 \pm 2.57$ & $5.23 \pm 1.34^{\mathrm{a}}$ & $3.16 \pm 1.54^{\mathrm{a}, \mathrm{b}}$ & $2.53 \pm 0.96^{\mathrm{a}-\mathrm{c}}$ & 22.367 & $<0.001$ \\
\hline $\mathrm{t}$ & 0.135 & 11.150 & 16.470 & 18.250 & & \\
\hline P-value & 0.892 & $<0.001$ & $<0.001$ & $<0.001$ & & \\
\hline
\end{tabular}

${ }^{\mathrm{a}} \mathrm{P}<0.05$, compared with the data before treatment; ${ }^{\mathrm{b}} \mathrm{P}<0.05$, compared with the data 1 month after treatment; ${ }^{\mathrm{c}} \mathrm{P}<0.05$, compared with the data 3 months after treatment. Marked response, the decrease of NIHSS score was $\geq 45 \%$ compared with that before treatment. Moderate response, the decrease of NIHSS score $<45 \%$ and $\geq 18 \%$ compared with that before treatment. No response, the decrease of NIHSS score $<18 \%$.

\section{Results}

General clinical data of patients in the control and observation groups. As shown in Table III, no significant difference was observed between the control and the observation group in terms of age, sex, disease type, low-density lipoprotein levels, hematological disease, fibrinogen, triglyceride and total cholesterol levels and alcohol abuse $(\mathrm{P}>0.05)$.

Comparison of neurological deficits and vascular recanalization between the two groups. NHISS scores before treatment were not statistically different between the two groups $(\mathrm{P}>0.05$; Table IV). At 1, 3 and 6 months after treatment, the NHISS scores were lower in the observation group than those in the control group $(\mathrm{P}<0.001)$. In the control group, the NIHSS scores at 1, 3 and 6 months after treatment were significantly lower than those before treatment $(\mathrm{P}<0.05)$; the NIHSS score at 1 month after treatment was not significantly different from that at 3 months after treatment; however, it was statistically different from that at 6 months after treatment $(\mathrm{P}<0.05)$; the NIHSS score 3 months after treatment was not different from that at 6 months after treatment. In the observation group, NIHSS scores at 1, 3 and 6 months after the treatment were significantly lower those before treatment and those in the control group after treatment $(\mathrm{P}<0.05)$; the NIHSS score at 1 month after treatment was statistically different from that at 3 and 6 months after treatment (both $\mathrm{P}<0.05$ ). As presented in Table $\mathrm{V}$, in the control group, there were 21 cases with complete recanalization, 22 cases with partial recanalization and 22 cases with no recanalization. In the observation group, there were 27 cases with complete recanalization, 23 cases with partial recanalization and 4 cases with no recanalization. 
Table V. Vascular recanalization after treatment in the control and observation groups [n (\%)].

\begin{tabular}{lcccr}
\hline Items & Control group $(\mathrm{n}=65)$ & Observation group $(\mathrm{n}=54)$ & $\chi^{2}$ & P-value \\
\hline Complete recanalization & $21(32.31)$ & $27(50.00)$ & 3.836 & 0.050 \\
Partial recanalization & $22(33.85)$ & $23(42.59)$ & 0.960 & 0.327 \\
Blocked & $22(33.85)$ & $4(7.41)$ & 12.07 & $<0.001$ \\
Total vascular recanalization & $43(66.15)$ & $50(92.59)$ & 12.07 & $<0.001$ \\
\hline
\end{tabular}

Table VI. Total effective rate after treatment in the control and observation groups [n (\%)].

\begin{tabular}{lcccc}
\hline Items & Control group $(\mathrm{n}=65)$ & Observation group $(\mathrm{n}=54)$ & $\chi^{2}$ & P-value \\
\hline Marked response & $23(35.38)$ & $25(46.30)$ & 1.459 & 0.227 \\
Moderate response & $21(32.31)$ & $23(42.59)$ & 1.339 & 0.247 \\
No response & $21(32.31)$ & $6(11.11)$ & 9.429 & 0.002 \\
Total effective rate & $44(67.69)$ & $48(88.89)$ & 9.429 & 0.002 \\
\hline
\end{tabular}

Marked response, $\geq 45 \%$ decrease of NIHSS score compared with that before treatment; moderate response, $<45 \%$ and $\geq 18 \%$ decrease of NIHSS score compared with that before treatment; no response, $<18 \%$ decrease of NIHSS score compared with that before treatment.

Table VII. Comparison of complications after treatment between the control and the observation group [n (\%)].

\begin{tabular}{lcccr}
\hline Complications & Control group $(\mathrm{n}=65)$ & Observation group $(\mathrm{n}=54)$ & $\chi^{2}$ & P-value \\
\hline Intracranial hemorrhage & $10(15.38)$ & $2(3.70)$ & 4.439 & 0.035 \\
Airway obstruction & $19(29.23)$ & $7(12.96)$ & 4.571 & 0.033 \\
Revascularization & $18(27.69)$ & $6(11.11)$ & 5.037 & 0.025 \\
Arrhythmia & $16(24.62)$ & $4(7.41)$ & 6.247 & 0.012 \\
\hline
\end{tabular}

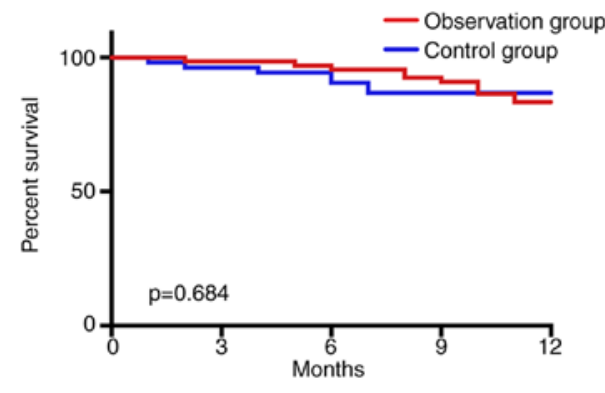

Figure 1. One-year survival curves of patients in the observation and control groups.

Total vascular recanalization was significantly better in the observation group than that in the control group $(\mathrm{P}<0.001)$.

One-year survival rate of the patients in the two groups after treatment. As shown in Fig. 1, all 119 patients were successfully followed up after treatment to assess 1-year survival. In the control group, 55 patients survived and 10 patients died within 1 year after treatment, with a survival rate of $84.62 \%$. In the observation group, 46 patients survived and 8 patients died within 1 year after treatment, with a survival rate of $85.19 \%$. According to the Kaplan-Meier survival curve analysis,

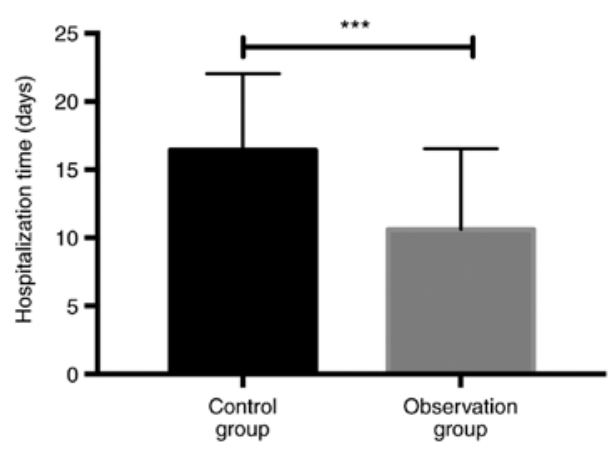

Figure 2. Comparison of total hospitalization time between the two groups. The total hospitalization time in the control group was statistically longer than that in the observation group $\left(\mathrm{t}=5.480,{ }^{* * *} \mathrm{P}<0.001\right)$.

the 1-year survival rate after treatment was not statistically different between the two groups $(\mathrm{P}=0.684)$.

Total effective rate after treatment in the control and observation groups. The rate of marked response was $46.30 \%$ in the observation group and $35.38 \%$ in the control group (Table VI). The rate of moderate response was $42.59 \%$ in the observation group and $32.31 \%$ in the control group. The rate of no response was $11.11 \%$ in the observation group and $32.31 \%$ in 
the control group. The observation group had a total effective rate of $88.89 \%$, significantly higher than the total effective rate $67.69 \%$ in the control group $\left(\chi^{2}=9.429, \mathrm{P}=0.002\right)$.

Comparison of complications after treatment between the control and observation groups. As presented in Table VII, in the observation group, 2 patients had intracranial hemorrhage after treatment, 7 airway obstruction, 6 revascularization and 4 arrhythmia. In the control group, 10 patients had intracranial hemorrhage after treatment, 19 airway obstruction, 18 revascularization and 16 arrhythmia. The complication rate in the observation group was statistically lower than that of the control group $(\mathrm{P}<0.05)$.

Comparison of total hospitalization time between the two groups. As shown in Fig. 2, the total hospitalization time in the control group was $16.42 \pm 5.62$ days, statistically longer than that in the observation group which was $10.62 \pm 5.90$ days $(\mathrm{t}=5.480, \mathrm{P}<0.001)$.

\section{Discussion}

ICD is a disease in which cerebrovascular wall lesions affect the supply of cerebral blood vessels, damaging the brain tissue of patients to varying degrees, and seriously affecting the patients' survival and quality of life (6). The impact of ICD on the nerve function and physical function of patients aggravate the economic burden of the patients and their families. Therefore, the search for an effective and safe treatment for ICD is of great significance (15). Currently, intravenous thrombolysis is the dominant therapy for treating ICD (16-18). However, only a limited number of patients are clinically suitable for intravenous thrombolysis therapy. As studies on cerebrovascular diseases go deeper, the minimally invasive neurointervention therapy begins to play a role in the treatment of cerebrovascular diseases (19-21). Neurointervention, a safe and highly efficient treatment, with fewer complications and less damage to the body functions, has started to be implemented in clinical practice (22).

The present study used the NIHSS score to assess the neurological function of patients. The NIHSS scores of the two groups after treatment were lower than those before treatment, and the NIHSS scores of patients treated with intravenous thrombolysis combined with neurointervention were significantly lower than those treated with intravenous thrombolysis only. The clinical treatment efficacy between the two groups was also compared. The total effective rate, complications, survival, and vascular recanalization for patients treated with intravenous thrombolysis combined with neurointervention were better than those treated with intravenous thrombolysis alone. A previous study (11) reported that neurointervention therapy brought a low incidence of complications and a high recanalization rate. The present study made further progress by combining intravenous thrombolysis with neurointervention, showing that intravenous thrombolysis combined with neurointervention can significantly reduce complications and improve the revascularization of patients with ICD, with better efficacy. The speculation is that the high selectivity of neurointervention compensates for the unspecified targets of intravenous thrombolysis. Moreover, intravenous thrombolysis can improve the problem of thrombus shedding resulted from microcirculation disturbance aroused by neurointervention. Therefore, the combination of neurointervention and intravenous thrombolysis has better efficacy, with less bleeding and side effects. Hao et al (23) explored the clinical effects of interventional therapy in ICD and showed that the combination therapy brought better treatment outcomes than the single use of drugs. Such a result is similar to our findings of the combination of neurointervention and intravenous thrombolysis in treating ICD. In the present study, the total hospitalization time after treatment in the observation group was significantly shorter than that in the control group. As reported by Coutinho et al (24), intravenous thrombolysis combined with thrombectomy was more effective than intravenous thrombolysis alone, which was similar to the result of the present study, i.e., that the efficacy of the combination therapy was better than that of the intravenous thrombolysis alone. In the present study, the results revealed that the combination therapy was more efficient leading to reduced hospitalization time. The combination of intravenous thrombolysis and mechanical thrombectomy used in Coutinho's et al study is defective because of the difficulty of the mechanical thrombectomy to be performed in the tortuous blood vessels. The neurointervention therapy can directly clear the blood vessels through arterial and venous intubation, resulting in high recanalization rates, better safety and treatment efficacy. According to the assessment of the patients' 1-year survival, the survival rate of the observation group was $85.19 \%$, whereas that of the control group was $84.62 \%$. No statistical difference was observed in the survival rate between the two groups. Although the effect of intravenous thrombolysis combined with neurointervention is better than that of intravenous thrombolysis alone, this combination can only recanalize the established thrombus. Numerous patients with ICD have high levels of blood lipids, which makes platelets easy to agglutinate into new thromboses leading to the recurrence of the disease. Unavailability of timely treatment in case of disease recurrence may lead to patient death. Therefore, these two treatment methods have no effect on the patient's survival.

The present study has some limitations. First, the time for the observation of the efficacy and adverse reactions was too short to get a clear understanding of the prognosis of patients, which should be improved in future studies. Second, other treatment methods were not explored for ICD, which are also needed to be discussed in subsequent studies. Finally, healthy individuals were not included in the study, making it unclear whether patients after treatment present differences from healthy subjects in some aspects. In future studies, healthy subjects will be enrolled, the follow-up time will be prolonged, and other treatment options will be investigated to supplement the deficiencies of the present study.

In conclusion, neurointervention combined with intravenous thrombolysis for treating ICD can reduce NHISS score. In addition, this combination can improve the recanalization rate, reduce complications and shorten the hospitalization time, achieving a higher effective rate.

\section{Acknowledgements}

Not applicable. 


\section{Funding}

No funding was received.

\section{Availability of data and materials}

The datasets used and/or analyzed during the current study are available from the corresponding author on reasonable request.

\section{Authors' contributions}

SL wrote the manuscript. SL and XL interpreted and analyzed the patient data. ZC and CS designed the study and performed the experiment. HL and $\mathrm{JH}$ were responsible for the analysis and discussion of the data. All authors read and approved the final manuscript.

\section{Ethics approval and consent to participate}

The present study was approved by the Ethics Committee of Xinxiang Central Hospital (Xinxiang, China). Patients who participated in the study had complete clinical data. Signed written informed consents were obtained from the patients and/or guardians.

\section{Patient consent for publication}

Not applicable.

\section{Competing interests}

The authors declare that they have no competing interests.

\section{References}

1. Powers WJ: Cerebral hemodynamics in ischemic cerebrovascular disease. Ann Neurol 29: 231-240, 1991.

2. Singh N, Moody AR, Gladstone DJ, Leung G, Ravikumar R, Zhan J and Maggisano R: Moderate carotid artery stenosis: MR imaging-depicted intraplaque hemorrhage predicts risk of cerebrovascular ischemic events in asymptomatic men. Radiology 252: 502-508, 2009.

3. Chen PP, Lou PA, Zhang P, Qiao C, Li T and Dong ZM: Trend analysis on the death rate of ischemic heart disease and cerebrovascular disease among Xuzhou residents from 2011 to 2015. Zhonghua Xin Xue Guan Bing Za Zhi 45: 597-607, 2017 (In Chinese).

4. Li X, Zhang ZW, Wang Z, Li JQ and Chen G: The role of argon in stroke. Med Gas Res 8: 64-66, 2018.

5. Sviridova NK and Yavorsky VV: The effectiveness of diagnosis and treatment of cerebrovascular disorders in hypertensive encephalopathy in elderly patients by studying the characteristics of cerebral hemodynamics and cerebral perfusion status. Lik Sprava: 41-46, 2015 (In Ukrainian).

6. Liu J, Liu Y, Wang L, Yin P, Liu S, You J, Zeng X and Zhou M: The disease burden of cardiovascular and circulatory diseases in China, 1990 and 2010. Zhonghua Yu Fang Yi Xue Za Zhi 49: 315-320, 2015 (In Chinese).

7. Zivanovic Z, Gubi M, Vlahovic D, Milicevic M, Jovicevic M, Lucic A, Ruzicka-Kaloci S, Radovanovic B, Zikic TR, Zarkov M, et al: Patients with acute lacunar infarction have benefit from intravenous thrombolysis. J Stroke Cerebrovasc Dis 28: 435-440, 2019.

8. Wu C, Wu D, Chen J, Li C and Ji X: Why not intravenous thrombolysis in patients with recurrent stroke within 3 months? Aging Dis 9: 309-316, 2018.
9. Fan Y, Liao X, Pan Y, Dong K, Wang Y and Wang Y; Thrombolysis Implementation and Monitor of Acute Ischemic Stroke in China (TIMS-China) Investigators: Intravenous thrombolysis is safe and effective for the cryptogenic stroke in China: Data from the thrombolysis implementation and monitor of acute ischemic stroke in China (TIMS-China). J Stroke Cerebrovasc Dis 28: 220-226, 2019

10. Wang MD, Yin XX, Yang TT, Wang Y, Zhu YY, Zhou YF, Lu ZX and Hu B: Chinese neurologists' perspective on intravenous thrombolysis for acute ischemic stroke. Brain Behav 8: $\mathrm{e} 00882,2017$

11. Mehta B, Leslie-Mazwi TM, Chandra RV, Chaudhry ZA, Rabinov JD, Hirsch JA, Schwamm LH, Rost NS and Yoo AJ: Assessing variability in neurointerventional practice patterns for acute ischemic stroke. J Neurointerv Surg 5 (Suppl 1): i52-i57, 2013.

12. Sacks D, Baxter B, Campbell BCV, Carpenter JS, Cognard C, Dippel D, Eesa M, Fischer U, Hausegger K, Hirsch, et al: Multisociety consensus quality improvement revised consensus statement for endovascular therapy of acute ischemic stroke: From the American Association of Neurological Surgeons (AANS), American Society of Neuroradiology (ASNR), Cardiovascular and Interventional Radiology Society of Europe (CIRSE), Canadian Interventional Radiology Association (CIRA), Congress of Neurological Surgeons (CNS), European Society of Minimally Invasive Neurological Therapy (ESMINT), European Society of Neuroradiology (ESNR), European Stroke Organization (ESO), Society for Cardiovascular Angiography and Interventions (SCAI), Society of Interventional Radiology (SIR), Society of NeuroInterventional Surgery (SNIS), and World Stroke Organization (WSO). Int J Stroke 13: 612-632, 2018.

13. Blackham KA, Meyers PM, Abruzzo TA, Albuquerque FC, Fiorella D, Fraser J, Frei D, Gandhi CD, Heck DV, Hirsch JA, et al: Endovascular therapy of acute ischemic stroke: Report of the standards of practice committee of the society of neurointerventional surgery. J Neurointerv Surg 4: 87-93, 2012.

14. Fischer U, Arnold M, Nedeltchev K, Brekenfeld C, Ballinari P, Remonda L, Schroth G and Mattle HP: NIHSS score and arteriographic findings in acute ischemic stroke. Stroke 36: 2121-2125, 2005.

15. Bondonno CP, Blekkenhorst LC, Prince RL, Ivey KL, Lewis JR, Devine A, Woodman RJ, Lundberg JO, Croft KD, Thompson PL and Hodgson JM: Association of vegetable nitrate intake with carotid atherosclerosis and ischemic cerebrovascular disease in older women. Stroke 48: 1724-1729, 2017.

16. Ishida J, Konishi M, Ebner N and Springer J: Repurposing of approved cardiovascular drugs. J Transl Med 14: 269, 2016.

17. Cheng NT and Kim AS: Intravenous thrombolysis for acute ischemic stroke within $3 \mathrm{~h}$ versus between 3 and $4.5 \mathrm{~h}$ of symptom onset. Neurohospitalist 5: 101-109, 2015.

18. Escudero D, Molina R, Viña L, Rodriguez P, Marqués L, Fernandez E, Forcelledo L, Otero J, Taboada F, Vega P, et al: Endovascular treatment and intra-arterial thrombolysis in acute ischemic stroke. Med Intensiva 34: 370-378, 2010 (In Spanish).

19. Rahman WT, Griauzde J, Chaudhary N, Pandey AS, Gemmete JJ and Chong ST: Neurovascular emergencies: Imaging diagnosis and neurointerventional treatment. Emerg Radiol 24: 183-193, 2017.

20. Yang Y, Liang C, Shen C, Tang H, Ma S, Zhang Q, Gao M, Dong Q and Xu R: The effects of pharmaceutical thrombolysis and multi-modal therapy on patients with acute posterior circulation ischemic stroke: Results of a one center retrospective study. Int J Surg 39: 197-201, 2017.

21. Radnai P, Szőts M, Rádai F, Horváth G, Varga C, Fogas J, Szörényi P, Horváth Z, Bajzik G, Moizs M, et al: Neurointerventional treatment of acute ischemic stroke: Our experience in kaposvar, Hungary. Ideggyogy Sz 68: 252-257, 2015 (In Hungarian).

22. Loftus CM, Hoffmann M, Heetderks W, Zheng X and Pena C: Regulation of neurological devices and neurointerventional endovascular approaches for acute ischemic stroke. Front Neurol 9: 320, 2018.

23. Hao Y, Qi Z, Ding Y, Yu X, Pang L and Zhao T: Effect of interventional therapy on IL-1 $\beta$, IL-6, and neutrophil-lymphocyte ratio (NLR) levels and outcomes in patients with ischemic cerebrovascular disease. Med Sci Monit 25: 610-617, 2019.

24. Coutinho JM, Liebeskind DS, Slater LA, Nogueira RG, Clark W, Dávalos A, Bonafé A, Jahan R, Fischer U, Gralla J, et al: Combined intravenous thrombolysis and thrombectomy vs thrombectomy alone for acute ischemic stroke: A pooled analysis of the SWIFT and STAR studies. JAMA Neurol 74: 268-274, 2017. 